\title{
Low-cost and Energy-efficient VLSI Architecture for Fast Integral Image Computing
}

\author{
Hao Xiao ${ }^{\mathrm{a},}$, Shu Chen ${ }^{\mathrm{b}}$, Da Shi ${ }^{\mathrm{c}}$ \\ ${ }^{a}$ Nanjing University of Aeronautics and Astronautics, Nanjing 210016, China \\ bShanghai Institute of Spaceflight Control Technology, Shanghai 201109, China \\ ' Jiangsu Yitong High-tech Co., Ltd., Changshu 215500, China
}

*Corresponding Author: xiaohao@nuaa.edu.cn

\begin{abstract}
This paper proposes a low-cost and energy-efficient VLSI architecture for fast integral image computing. The proposed architecture distributes large size image blocks onto multiple cascaded small size processing units, which enables the comprehensive and fine-grained optimization of the bit width of adders and registers used in the integral image computing. The proposed integral image engine is implemented in RTL and verified on a FPGA-based SoC platform for real-time human face detection. Compared with the state-of-the-art designs, the proposed architecture can reduce up to $33.6 \%$ gate count and $31.5 \%$ power dissipation.
\end{abstract}

Keywords: Integral image, human face detection, cascaded architecture, parallel processing.

\section{Introduction}

Integral image, which is also known as the summed-area table [1], is an important algorithm in the world of computer vision for quickly and effectively calculating the sum of pixel values in a given image. It was first introduced with the Viola-Jones Object Detection Framework [2], and since then, it has been commonly used in the fields of object detection, speech detection, and pedestrian recognition, especially for the fast calculation of Haar-like features [3-5]. Although the integral image is an effective way to quickly compute the features, the calculation process is computation and memory accessing intensive, and usually accounts for large parts of the total execution time. Thus, the implementation of fast integral image computing is of vital practical significance, especially for embedded applications where real-time processing is required within a constrained cost and power budget.

In order to quickly and efficiently compute the integral image in embedded systems, specialized hardware solution that consumes less power and area cost is considered to be the best alternative. For the Haar feature based face detection, Kimura [6] proposed a low power architecture and Hiromoto [7] proposed a partial configurable architecture. Both work achieve the integral computing by accumulating the image intensity one by one with the computation complexity of $O(m \times n)$, where $m$ and $n$ denote the number of columns and rows in an image, respectively. In addition, Kyrkou etc. [8] proposed a new hardware architecture of integral image computing with reduced computing complexity of $O(2 m+n)$, which largely improves the computing speed. More recently, the authors in $[9,10]$ exploit the parallelism of the integral image computing by proposing a dual-direction (row direction and column direction) data-oriented method to support the generic computing of integral image. This method reduces the time complexity for computing an $m \times n$ image to $O(m+$ $n$ ), and also designs a pipelined structure that further reduces the complexity to $O(n)$. However, one important disadvantage lies in the architecture of $[9,10]$ is that it simply gives all the adders and registers required for computing and storing the integral image values with the same maximum bit width, while lacking a fine-grained profiling of the required bit width at different pipeline stage. As the size of the input image grows, the adder and the storage grow proportionally, resulting in a significant 
hardware overhead.

Therefore, this paper attempts to optimize the dual-direction data-oriented method by proposing a new processing structure to improve its hardware efficiency. The contribution of this work comes twofold: (i) we propose a cascaded processing structure to distribute a large size image block onto multiple fine-grain processing units, whose bit width can be reduced without spoiling the accuracy; (ii) the proposed integral image engine is further wrapped as a functional IP and integrated into an FPGA-based SoC platform for real-time human face detection. We implement the proposed architecture using Verilog HDL and synthesize it using TSMC 90nm technology. For comparison, we also implement the integral image computing methods in $[9,10]$. Results show that the proposed architecture can reduce up to $33.6 \%$ gate count and $31.5 \%$ power dissipation.

The rest of this brief is organized as follows. Section 2 introduces the integral image algorithm, which is followed by the description of the proposed method in Section 3 . Hardware implementation and experimental results are presented in Section 4 and 5, respectively. Finally, conclusions are drawn in Section 6.

\section{Algorithm}

Integral image is a data structure that can quickly and efficiently compute the sum of pixel values in a rectangular subset of a grid [1]. As shown in Fig. 1(a), the integral image value $I I(x, y)$ at location $(x, y)$ is equal to the sum of the intensity of all pixels above and to the left of location ( $x$, $y$ ) in the original image $p(x, y)$. It's definition can be represented by the Eq. (1):

$$
I I(x, y)=\sum_{x^{\prime}<x} \sum_{y^{\prime}<y} p\left(x^{\prime}, y^{\prime}\right)
$$

The integral image has a useful property that can benefit its hardware implementation. As shown in Fig. 1(b), the image that starts at point $(0,0)$ and ends at point $\left(x_{0}, y_{0}\right)$ can be divided into two parts $\mathrm{A}$ and $\mathrm{B}$. Thus the integral image value $\operatorname{II}\left(x_{0}, y_{0}\right)$ at $\left(x_{0}, y_{0}\right)$ can be represented by the sum of the intensity of all the pixels in area A plus the sum of intensity of pixels in area B, which is shown in Eq. (2). Then, we can further get Eq. (3), where the integral image value $\operatorname{II}\left(x_{0}, y_{0}\right)$ at $\left(x_{0}, y_{0}\right)$ equals the integral image value at the point $\left(x_{1}, y_{0}\right)$ plus the sum of intensity of pixels in area $\mathrm{B}$. Thus, when computing the integral image values in $\mathrm{B}$,
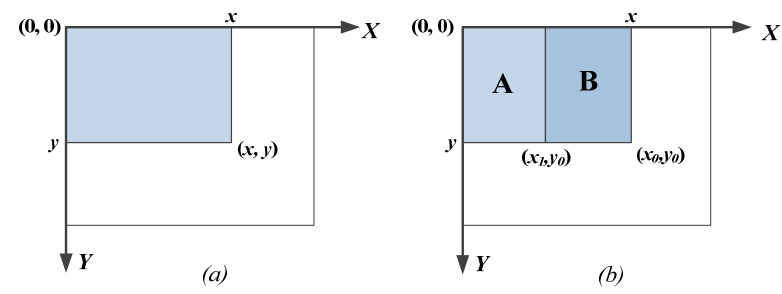

Fig. 1. (a) Definition of integral image, (b) A property of integral image.

we can regard B as an independent image and compute its integral image; then, we add each row of integral values with the rightmost value at the same row of A's integral image. In other word, we only need to know the value of the rightmost column in the integral image of A to compute the integral image value of $\mathrm{B}$.

$$
\begin{aligned}
& I I(x, y)=\sum_{x^{\prime}<x_{1}} \sum_{y^{\prime}<y_{0}} p\left(x^{\prime}, y^{\prime}\right)+\sum_{x_{1} \leq x^{\prime}<x_{1}} \sum_{y^{\prime}<y_{0}} p\left(x^{\prime}, y^{\prime}\right) \\
& I I(x, y)=I I\left(x_{1}, y_{0}\right)+\sum_{x_{1} \leq x^{\prime}<x_{1}} \sum_{y^{\prime}<y_{0}} p\left(x^{\prime}, y^{\prime}\right)
\end{aligned}
$$

\section{Proposed Method}

In [9], Peng etc. proposes a dual-direction data-oriented integral image computing method to exploit the parallelism. And in [10], they further optimize the architecture by reducing the pipeline depth of the processing unit. As shown in Fig. 2(a), this architecture employs a lot of adders and registers for achieving high parallelism. As described above, the integral image is the sum of the pixels in the original image, and hence, with the sum accumulating, these adders and registers need enough width to avoid overflow. For an $m \times n$ 8-bit grayscale image, the maximum bit width for one pixel required in its integral image is shown Equ. (4). However, one important disadvantage in [9] and [10] is that it simply gives all the adders and registers with the same maximum bit width, while lacking a fine-grained profiling of the required bit width at different pipeline stage. Obviously, this results in a significant hardware overhead, especially as the size of image increases.

$$
W=\left[\log _{2}(m \times n \times 255)\right]+1
$$

Therefore, in order to reduce this hardware redundancy, this paper proposes a method to provide a 


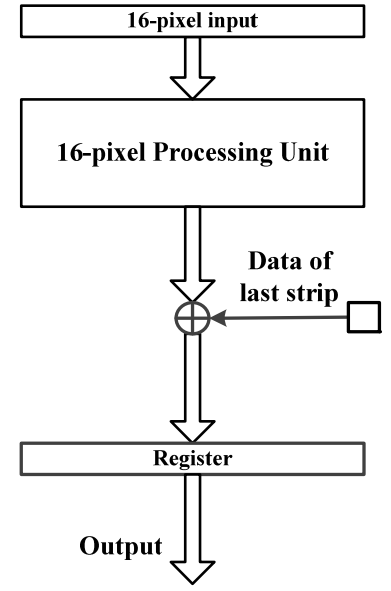

(a)

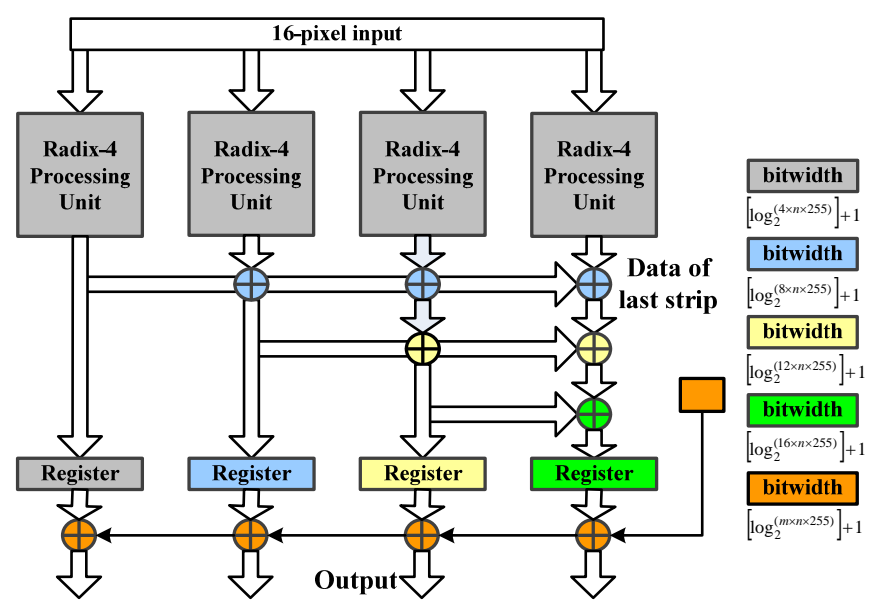

(b)

Fig. 2. (a) Stand-alone 16-pixel input processing array, (b) proposed cascaded processing array

Table 1. Comparison of hardware resource.

\begin{tabular}{|c|c|c|c|}
\hline W=16 & Ref. [9] & Ref. [10] & Proposed \\
\hline \#Adders & $47 \times W_{5}$ & $81 \times W_{5}$ & $36 \times W_{1}+12 \times W_{2}+8 \times W_{3}+4 \times W_{4}+16 \times W_{5}$ \\
\hline \#Registers & $272 \times W_{5}$ & $85 \times W_{5}$ & $52 \times W_{1}+4 \times W_{2}+4 \times W_{3}+4 \times W_{4}+1 \times W_{5}$ \\
\hline
\end{tabular}

comprehensive and fine-grained optimization of the bit width of adders and registers used in the integral image computing. According to the algorithm in section 2, the calculation of a large size integral image can be separated into several independent small ones. Therefore, unlike [9, 10] that use a stand-alone 32-pixel input processing array, this method uses a set of cascaded small size, for instance 4-pixel, processing units. The most important advantage brought by this method is that these small size processing units are independent and their maximum bit width required for avoiding overflow is much smaller, making it easy to tailor the required bit width for each unit with minimum hardware overhead. Fig. 2(b) illustrates the idea with a 16-pixel input example. According to the depth of the pipeline stage, we classify the bit width of the architecture into five fine-grained catalogues, where each catalogue can meet the maximum bit width requirement of the corresponding pipeline stage. Table 1 compares the hardware resource used in the proposed architecture with Ref. 9 and Ref. 10. As shown, the proposed architecture employs a similar amount of hardware resource, while reducing the bit width of the adders and registers, significantly.

\section{Hardware Architecture}

\subsection{Hardware Overview}

As shown in Fig. 3, The proposed architecture mainly consists of a control unit (CU), a calculation unit (CALU), an original image memory (OIM) and an integral image memory (IIM). During calculation, the pixel values of the original image is stored in OIM with its natural sequence, and then, fetched for calculation with a revised order generated by the CU. Then, the integral image is calculated by the CALU and the results are written into the integral image memory (IIM). On the other hand, the rightmost value of the previous strip that are already stored in IIM are also feedback to CALU for the calculation of the current strip. It is noted that we implement the proposed architecture in Xilinx FPGA, whose RAM block supports

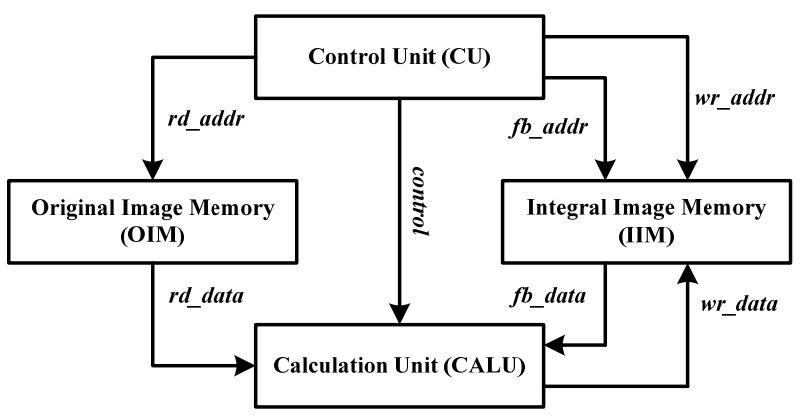

Fig. 3. System overview. 


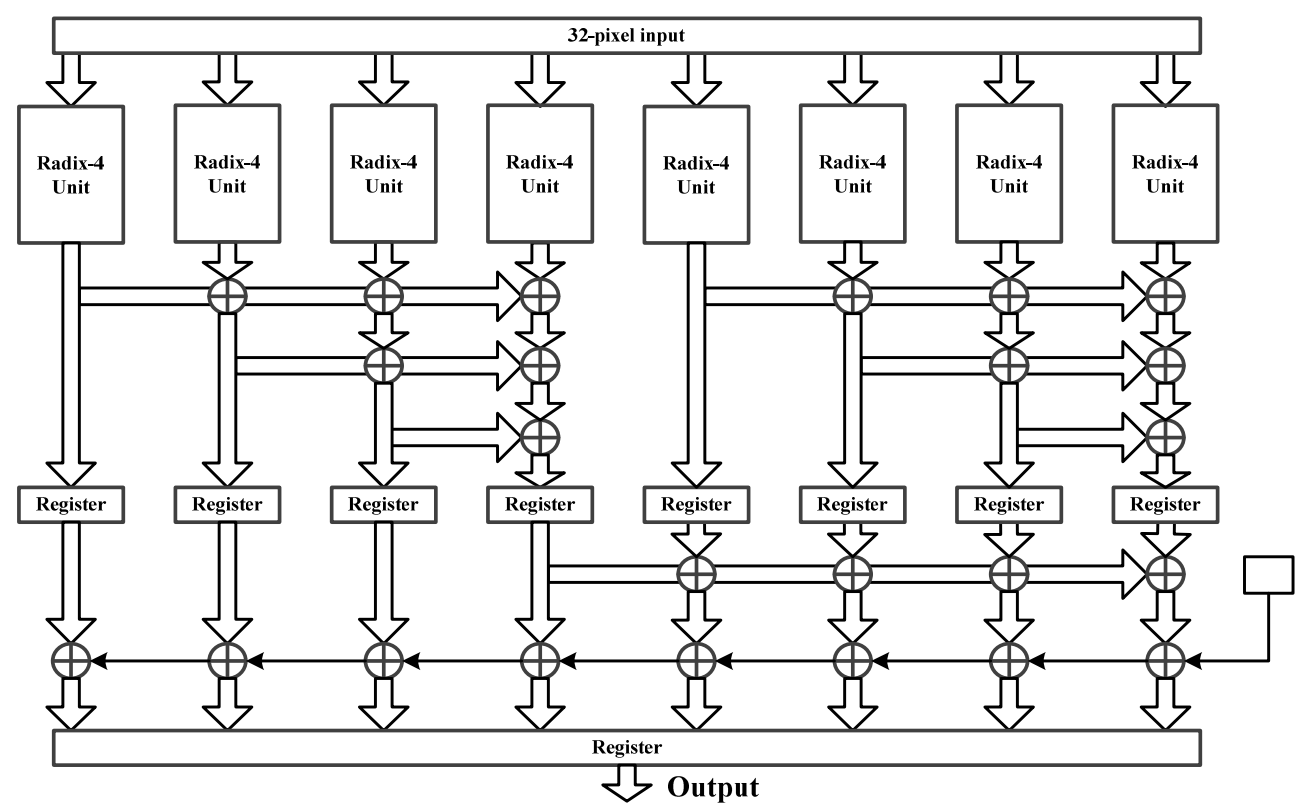

Fig. 4. Computation unit.
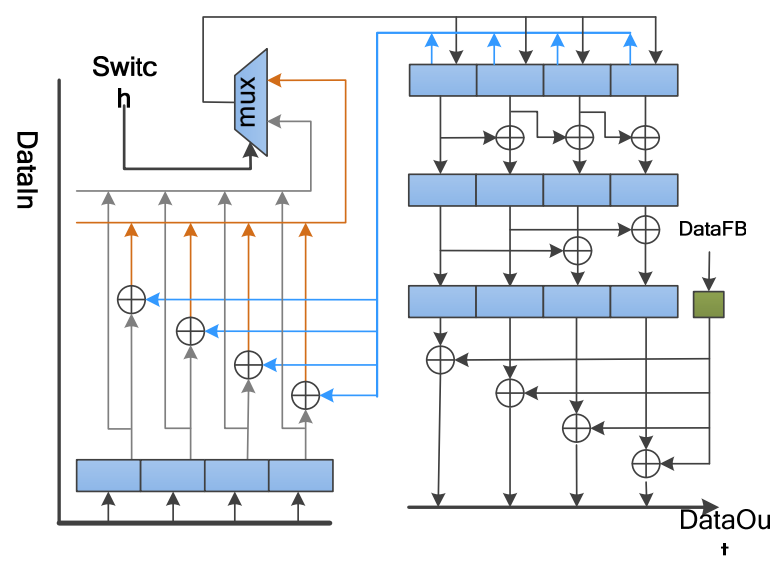

Fig. 5. Structure of Radix-4 computation unit.

bit width upto 4608 bits, making it enough to read/write a row of pixels of a strip in one cycle. Thus, unlike Ref. 9 and Ref. 10, we can get rid of the FIFO to buffer the results.

The whole calculation process of the proposed architecture follows the dual-direction data-oriented method. For an $m \times n$ image, it is divided into $m / w$ strips, where $w$ is the width of each strip (usually $m$ could be exactly divided by $w$ ). The image pixels are accessed row by row in each "strip" and "strip" by "strip". The original image is stored in RAM devices, whose interface width is the bit width of the row of a strip. Thus, a row of pixels of a strip could be accessed in one cycle where rows of the same "strip" are stored in continuous addresses and "strip" are stored one by one. The output integral image memory is also a RAM device, whose interface width is the bit width of the data output of CALU.

\subsection{Computation Unit}

In this work, a cascaded calculation unit is proposed to implement the integral image algorithm in a pipeline manner. According to the analysis in [9], using $w=32$ can achieve the best tradeoff between the hardware cost and energy efficiency. Thus, the proposed CALU also uses this configuration. As shown in Fig. 4, the CALU consists of eight cascaded Radix-4 integral unit, which are divided into two groups with four Radix-4 units for each. Each group is actually an independent 16-pixel input processing array, whose structure is shown in Fig. 2(b). Each Radix-4 unit calculates the integral value of a $4 \times n$ image strip independently. According to Eq. (3), the pixel value of the integral image can be represented by the sum of the current value and the result of the rightmost column of the last strip. Thus, after calculating the integral value of each independent $4 \times n$ image strip, the rightmost integral value of each Radix-4 unit is added up to all the results on its right side. Considering the length of the critical path, we let each group to perform the sum operation first. Then, in the following cycle, the rightmost value of the left group is added up to the results of the right group. Moreover, at the last stage, the rightmost integral value is loaded from IIM by CU, passing the data from right to left.

The Radix-4 integral unit, as shown in Fig. 5, is designed following the dual-direction (row direction and column direction) data-oriented method [10]. It can compute the integral image data of a $4 \times n$ image. It is noteworthy that one important advantage of the proposed 


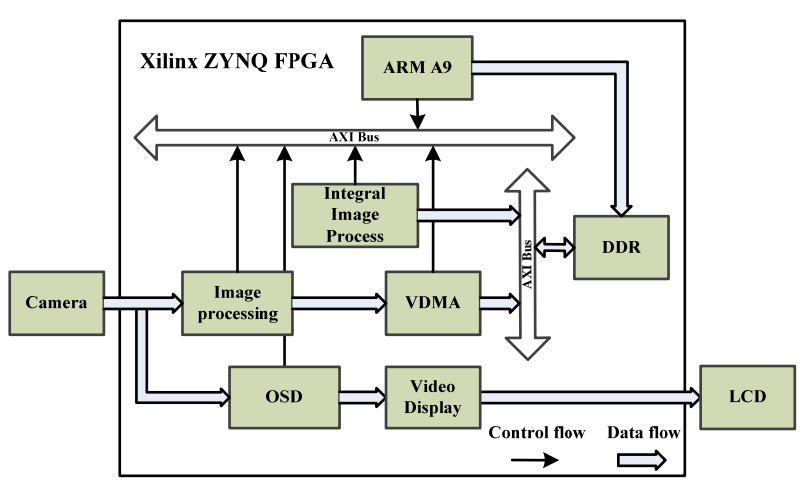

Fig. 6. SoC-based face detection system

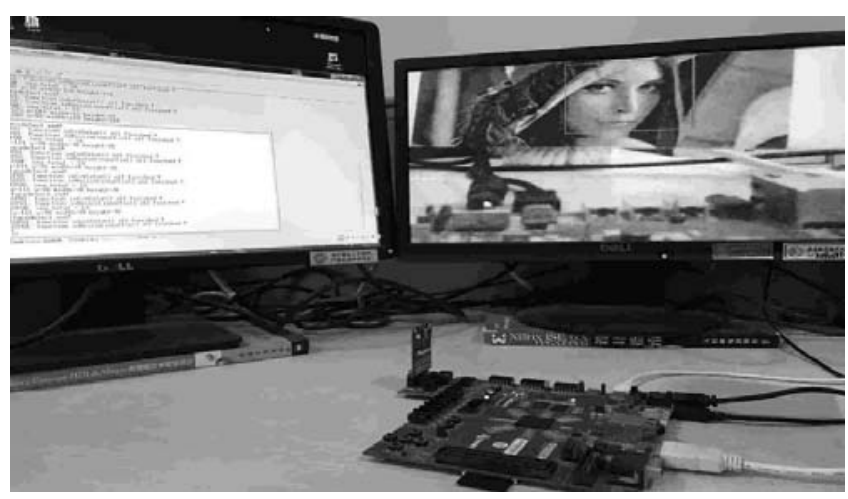

Fig. 7. Snapshot of the FPGA implementation platform.

architecture lies in the saving of bit width of the adders and registers required for computing the integral image values. Each Radix-4 integral unit carries out a $4 \times n$ integral computation in a pipeline way independently. Thus, considering the overflow of the addition, the width of the register and adder of the Radix-4 integral unit is fixed to be $\left[\log _{2}(4 \times n \times 255)\right]+1$ bit. Only the registers and adders outside Radix-4 processing units are necessary to progressively increase the bit width to avoid overflow.

\section{Implemantation and Result}

\section{$5.1 \quad$ System setup}

The proposed integral image engine is implemented using Verilog HDL and prototyped on FPGA. To verify the correctness of the implementation, we setup a stand-alone environment, where the integral image value of each image computed by our architecture are compared with the theoretical results computed by OpenCV's library. In addition, we also verify the proposed architecture in a complete AdaBoost face detection system, where the face detection algorithm is SW-implemented and the integral image computation, as well as image processing pipeline, are $\mathrm{HW}$-implemented to boost the performance. As shown in Fig. 6 and Fig. 7, we use Xilinx ZYNQ SoC platform to implement the face detection system. The SW part executes on the ARM A9 dual-core processor and the HW part is implemented on the FPGA. The input video is duplicated into two streams, one is stored into the DDR for face detection and the other is output directly for video display. To integrate the integral image engine with the face detection system, we employ a DMA engine, which on the one hand moves the pre-processed image into its original image RAM, and on the other hand transfers the processing results from the integral image RAM back to the DDR. Then, the SW further uses the integral image to perform the face detection and marks the detected position onto the original image via a common Video On-Screen Display (OSD) core.

\subsection{Performance evaluation}

To evaluate the area and power efficiency of the proposed architecture, we synthesize the design using TSMC 90nm library and targeting a $500 \mathrm{MHz}$ clock cycle.

Table 2. Comparison of gate count.

\begin{tabular}{|c|c|c|c|c|}
\hline & $4096 \times 2160$ & $1920 \times 1080$ & $1280 \times 720$ & $640 \times 480$ \\
\hline Proposed & 64331 & 57402 & 53876 & 53709 \\
\hline Ref. [10] & 96906 & 85520 & 78616 & 77021 \\
\hline Ref. [9] & 206217 & 184776 & 174577 & 169621 \\
\hline
\end{tabular}

Table 3. Comparison of dynamic power $(m W)$.

\begin{tabular}{|c|c|c|c|c|}
\hline & $4096 \times 2160$ & $1920 \times 1080$ & $1280 \times 720$ & $640 \times 480$ \\
\hline Proposed & 25.6 & 23.6 & 23.1 & 22.6 \\
\hline Ref. [10] & 37.4 & 33.7 & 32.5 & 31.4 \\
\hline Ref. [9] & 161.9 & 146.6 & 141.5 & 136.5 \\
\hline
\end{tabular}


For comparison, we also implement the architecture in [9] [10], and synthesize them with the same technology. Table 2 and Table 3 report the synthesis results at various resolution. In the architecture proposed in Ref. [9], the number of register is proportional to $w^{2}$, where $w$ is the width of each strip. Ref. [10] optimizes this structure by using more adders while reducing the number of registers significantly. Thus, as shown in the table, Ref. [10] costs much less gate count than Ref. [9]. However, compared with Ref. [10], the proposed architecture can further reduce up to $33.6 \%$ gate count and $31.5 \%$ power dissipation. More importantly, the proportion of area and power saving increases as the resolution increases, which meets our expectation in section 1. It is noted that the proposed architecture optimizes the bit width of adders and registers used in computing integral image without spoiling the performance and accuracy. Thus, although this architecture saves a lot of area and power, it can achieve the same performance as Ref. [10].

\section{Conclusions}

This paper proposes a hardware architecture for low-cost and energy-efficient integral image computing. To reduce hardware redundancy, this paper provides a comprehensive and fine-grained optimization of the bit width of adders and registers used in the integral image computing. By distributing large size image blocks onto multiple cascaded small size processing units, the proposed architecture makes it easy to tailor the required bit width for each processing unit. Then, according to the depth of the pipeline stage, we elaborately classify the bit width of the architecture into five fine-grained catalogues, and assign each pipeline stage with the minimum required bit width to reduce hardware overhead. Results show that, comparing to the state-of-the-art designs, the proposed architecture can reduce the area and power dissipation significantly.

\section{Acknowledgment}

This work is supported in part by National Natural Science Foundation of China 61504059, Natural Science Foundation of Jiangsu Province BK20140834, and Fundamental Research Funds for the Central Universities NS2015043.
(1) Franklin C. Crow: "Summed-Area Tables for Texture Mapping”, SIGGRAPH Proc., pp. 207-212, July 1984.

(2) P. Viola, M. Jones: "Rapid Object Detection using a Boosted Cascade of Simple Features", IEEE Conference on Computer Vision and Pattern Recognition, pp. 511 - 518, Dec. 2001.

(3) Y. Hanai, et al.: "A Versatile Recognition Processor Employing Haar-like Feature and Cascaded Classifier", IEEE International Solid-State Circuits Conference (ISSCC), pp. 148 - 149, February 2009.

(4) R. Miyamoto, et al.: "Pedestrian Recognition in Far-infrared Images by Combining Boosting-based Detection and Skeleton-based Stochastic Tracking", PSIVT Proc., pp. 483 - 494, 2006.

(5) T. Theocharides, et al.: "A parallel architecture for hardware face detection”, IEEE Comput. Soc. Annu. Symp. VLSI: Emerging VLSI Technologies Architectures, pp. 452 - 453, March 2006.

(6) M. Kimura, et al.: "A low-power AdaBoost-based object detection processor using Haar-like features", IEEE 3rd International Conference on Consumer Electronics, pp. 203 - 206, Sept. 2013.

(7) M. Hiromoto, et al.: "Partially parallel architecture for AdaBoost-based detection with Haar-like features", IEEE Transactions on Circuits and Systems for Video Technology, vol. 19, no. 1, pp. 41 - 52, Jan. 2009.

(8) C. Kyrkou, et al.: "A flexible parallel hardware architecture for AdaBoost-based real-time object detection”, IEEE Trans. Very Large Scale Integr. (VLSI) Syst., vol. 19, no. 6, pp. 1034 - 1047, June 2011.

(9) P Ouyang, S Yin, Y Zhang, et al.: “A Fast Integral Image Computing Hardware Architecture With High Power and Area Efficiency”, IEEE Transactions on Circuits \& Systems II Express Briefs, vol. 62, no. 1, pp. 75-79, Jan. 2015.

(10) Shouyi Yin, et al.: "A Configurable Parallel Hardware Architecture for Efficient Integral Histogram Image Computing”, IEEE Trans. Very Large Scale Integr. (VLSI) Syst, vol. 24, no. 4, pp. 1305-1318, April 2016.

\section{References}

